\title{
APROVEITAMENTO DA GLICERINA ORIUNDA DO BIODIESEL NO CULTIVO DE Cryptococcus curvatus NRRL Y- 1511 PARA PRODUÇÃO DE LIPASE
}

\author{
B.L. LADEIRA ${ }^{1}$ e C.A.V.BURKERT ${ }^{1}$ \\ ${ }^{1}$ Universidade Federal do Rio Grande. Escola de Química e Alimentos. \\ E-mail para contato: profbrunoladeira@gmail.com
}

RESUMO - Este trabalho teve como objetivo investigar o uso da glicerina residual no cultivo de Cryptococcus curvatus NRRL Y-1511 para produção de lipase. Para o cultivo microbiano foi usado o meio de cultura contendo glicerina residual, extrato de malte, extrato de levedura, peptona e Tween 80 , sendo retiradas alíquotas dos cultivos para a determinação da biomassa máxima, produtividade em biomassa, velocidade específica máxima de crescimento celular, atividade lipolítica e produtividade enzimática. Para maximizar a produção da enzima, um planejamento fracionário $2 \mathrm{IV}^{7-3}$ e um planejamento completo $2^{3}$ foram realizados sequencialmente. As condições estabelecidas para a produção de lipase foram: glicerol $(17,5$ g. $\left.\mathrm{L}^{-1}\right)$; extrato de levedura $\left(3\right.$ g.L. $\left.\mathrm{L}^{-1}\right)$; extrato de malte $\left(3 \mathrm{~g} . \mathrm{L}^{-1}\right)$; peptona $\left(5\right.$ g.L $\left.\mathrm{L}^{-1}\right)$; Tween 80 $\left(7,5\right.$ g. $\left.\mathrm{L}^{-1}\right)$; temperatura $\left(30^{\circ} \mathrm{C}\right) ; \mathrm{pH}(6,0)$ e $24 \mathrm{~h}$ de cultivo. Nestas condições atingiu-se uma atividade lipolítica máxima de $2,15 \mathrm{U} \cdot \mathrm{mL}^{-1}$, uma biomassa máxima de $12,46 \mathrm{~g} . \mathrm{L}^{-1}$, uma produtividade de biomassa de $0,20 \mathrm{~g} \cdot \mathrm{L}^{-1} \cdot \mathrm{h}^{-1}$, velocidade especifica máxima de crescimento celular de $0,343 \mathrm{~h}^{-1}$ e produtividade enzimática de $0,094 \mathrm{U} \cdot \mathrm{mL}^{-1} \cdot \mathrm{h}^{-1}$.

\section{INTRODUÇÃO}

Nas ultimas décadas, tem-se observado uma intensiva busca por fontes alternativas de energia e processos sustentáveis visando solucionar a problemática da crise energética, bem como a redução da poluição ambiental e o aquecimento global do planeta (Bilgen et al., 2008). Os biocombustíveis, como o biodiesel, representam uma alternativa renovável e ambientalmente segura aos combustíveis fósseis, visto que além de ajudar a diminuir as emissões de gases de efeito estufa que gera um grande impacto negativo sobre a biodiversidade é ao mesmo tempo uma fonte estratégica de energia renovável para substituir o óleo diesel e derivados de petróleo (Accarini, 2009).

No cenário brasileiro, a literatura reporta que por questões de cunho cultural e territorial o Brasil é o maior produtor e ao mesmo tempo consumidor mundial de biodiesel, uma vez que possui grande disponibilidade de matéria-prima e um crescimento contínuo na indústria de óleos vegetais e do etanol (Silva et al., 2009).

Na conjuntura atual, conforme dados da ANP, a quantidade de biodiesel produzida em todo território brasileiro no ano de 2012 foi de $2.717 .438 \mathrm{~m}^{3}$ e de $2.917 .418 \mathrm{~m}^{3}$ em 2013 . No estado do Rio Grande do Sul em 2012 foram produzidos cerca de $806.500 \mathrm{~m}^{3}$ enquanto que 
em 2013 foram produzidos $883.331 \mathrm{~m}^{3}$. Neste cenário, um fator preocupante é a geração da glicerina bruta como subproduto em proporção apreciável (cerca de 10\%), podendo causar danos ao meio ambiente e encarecer a produção do biodiesel (Silva et al., 2009).

Segundo a Associação Brasileira da Indústria química a demanda pela glicerina bruta é bem menor do que a sua produção e os mercados estão encontrando dificuldades em absorver essa nova oferta de produto. Desta forma, muitas pesquisas são conduzidas em busca de novas aplicações para o excesso de glicerina bruta. Uma alternativa promissora está no estudo da conversão microbiana da glicerina em produtos de maior valor agregado (Hasan et al., 2006; Rivaldi et al., 2007).

Neste contexto, as lipases de origem microbiana são biomoléculas atraentes, são produzidas por ampla faixa de micro-organismos, apresentam custo de produção relativamente baixo e reúnem uma série de propriedades que as tornam versáteis industrialmente, visto que catalisam inúmeras reações diferentes de bioconversão, tais como: hidrólise, inter esterificação, esterificação, alcoólise e acidólise (Treichel et al., 2010 ; Lee et al., 2007).

\section{MATERIAL E MÉTODOS}

Os cultivos da levedura Cryptococcus curvatus NRRL Y-1511 foram realizados em frascos Erlenmeyer de $500 \mathrm{~mL}$ contendo $200 \mathrm{~mL}$ de meio com a composição e pH inicial estabelecidos de acordo com o planejamento experimental. Os frascos foram inoculados com suspensão de leveduras previamente preparada de forma a atingir concentração celular inicial de $1 \times 10^{7}$ células. $\mathrm{mL}^{-1}$. A quantidade de glicerina bruta adicionada ao meio de cultivo levou em conta sua composição ( $83 \%$ de glicerol) a fim de resultar na concentração de glicerol desejada nos meios de cultivo.

Os frascos foram mantidos em incubadora rotatória com temperatura controlada e 180 rpm de agitação, retirou-se alíquotas ao longo do cultivo, sendo estas centrifugadas sob refrigeração $\left(7^{\circ} \mathrm{C}\right)$ a 5241 x g por 15 min para as determinações analíticas no sobrenadante e no sedimento.

O crescimento celular foi monitorado por medida da absorbância a $600 \mathrm{~nm}$. A concentração de biomassa foi expressa em massa seca $\left(\mathrm{g} \cdot \mathrm{L}^{-1}\right)$, a partir de uma curva padrão de biomassa determinada para Cryptococcus curvatus NRRL Y-1511. Com a biomassa foram também obtidos dados relativos à produtividade em biomassa, em g. $\mathrm{L}^{-1} \cdot \mathrm{h}^{-1}$ e velocidade específica máxima de crescimento celular, em $\mathrm{h}^{-1} . \mathrm{O} \mathrm{pH}$ ao longo dos cultivos foi medido diretamente em medidor de $\mathrm{pH}$ previamente calibrado (AOAC, 2000).

Para determinação da atividade lipolítica foi adotado o método baseado na titulação dos ácidos graxos liberados pela ação da enzima lipase, presente no caldo bruto livre de células, sobre os triacilgliceróis do óleo de oliva emulsionados em goma arábica, conforme descrito por Burkert (2003). A atividade lipolítica foi calculada através da equação 1. Foi também determinada a produtividade enzimática através da equação 2. 


$$
\begin{aligned}
& A t i v=\frac{\Delta V \cdot N \cdot 1000}{t \cdot v} \\
& \operatorname{Prod}_{E}=\frac{A t i v_{\max }}{t_{f}}
\end{aligned}
$$

Onde:

Ativ: Atividade lipolítica $\left(\mathrm{U} \cdot \mathrm{mL}^{-1}\right)$;

Ativ $v_{\max }$ : Atividade lipolítica máxima alcançada em cada ensaio $\left(\mathrm{U} \cdot \mathrm{mL}^{-1}\right)$;

Prode: Produtividade enzimática $\left(\mathrm{U}_{\mathrm{E}} \mathrm{mL}^{-1} \cdot \mathrm{h}^{-1}\right)$;

$\Delta \mathrm{V}$ : Diferença entre o volume gasto para titular a amostra e o branco $(\mathrm{mL})$;

v: Volume de caldo bruto livre de células $(\mathrm{mL})$;

$\mathrm{N}$ : Normalidade da solução de hidróxido de sódio $(\mathrm{N})$;

t: Tempo de reação $(\min )$;

$\mathrm{t}_{\mathrm{f}}$ : Tempo de cultivo (h);

Com a levedura Cryptococcus curvatus NRRL Y-1511 foi proposto um primeiro planejamento experimental fracionário $2^{7-3}$ de resolução IV para avaliar os efeitos da temperatura, $\mathrm{pH}$ e concentrações de glicerol, extrato de levedura, extrato de malte, peptona e Tween 80 sobre a produção de lipase em frascos agitados. Os valores reais e codificados das variáveis deste planejamento estão apresentados na Tabela 1.

Tabela 1 - Faixa de valores estudados no planejamento experimental $2^{7-3}$.

\begin{tabular}{cccccccc}
\hline Nível & $\begin{array}{c}\text { Glicerol } \\
\left(\mathrm{g} . \mathrm{L}^{-1}\right)\end{array}$ & $\begin{array}{c}\text { Tween } 80 \\
\left(\mathrm{~g} . \mathrm{L}^{-1}\right)\end{array}$ & $\begin{array}{c}\text { Extrato de } \\
\text { levedura } \\
\left(\mathrm{g} . \mathrm{L}^{-1}\right)\end{array}$ & $\begin{array}{c}\text { Extrato } \\
\text { de malte } \\
\left(\mathrm{g} . \mathrm{L}^{-1}\right)\end{array}$ & $\begin{array}{c}\text { Peptona } \\
\left(\mathrm{g} . \mathrm{L}^{-1}\right)\end{array}$ & $\mathrm{pH}$ & $\mathrm{T}\left({ }^{\circ} \mathrm{C}\right)$ \\
\hline-1 & 5 & 0 & 1 & 1 & 1 & 5 & 25 \\
0 & 10 & 5 & 3 & 3 & 5 & 6 & 30 \\
+1 & 15 & 10 & 5 & 5 & 9 & 7 & 35 \\
\hline
\end{tabular}

Com as variáveis mais influentes selecionadas no planejamento fracionário $2^{7-3}$ foi proposto um DCCR completo $2^{3}$ visando a otimização da composição do meio de cultivo para a maximização da atividade lipolítica. Os valores reais e codificados das variáveis utilizadas neste planejamento estão apresentados na Tabela 2.

Tabela 2 - Faixa de valores estudados no DCCR completo $2^{3}$

\begin{tabular}{cccc}
\hline Nivel & $\mathrm{T}\left({ }^{\circ} \mathrm{C}\right)$ & Tween $80\left(\mathrm{~g} \cdot \mathrm{L}^{-1}\right)$ & Glicerol $\left(\mathrm{g} . \mathrm{L}^{-1}\right)$ \\
\hline$-1,68$ & 25 & 2,5 & 7,5 \\
-1 & 27 & 4,5 & 9,5 \\
0 & 30 & 7,5 & 12,5 \\
+1 & 33 & 10,5 & 15,5 \\
$+1,68$ & 35 & 12,5 & 17,5 \\
\hline
\end{tabular}




\section{RESULTADOS E DISCUSSÃO}

\subsection{Planejamento experimental fracionário}

A Tabela 3 apresenta a matriz do planejamento fracionário $2^{7-3}$, com as variáveis estudas e respectivas respostas com o objetivo de selecionar as variáveis mais influentes para posterior planejamento completo e consequente otimização do meio de cultivo.

Tabela 3- Matriz do planejamento fracionário $2^{7-3}$ e respostas obtidas.

\begin{tabular}{ccccccccccccc}
\hline Ensaio & $\mathrm{X}_{1}$ & $\mathrm{X}_{2}$ & $\mathrm{X}_{3}$ & $\mathrm{X}_{4}$ & $\mathrm{X}_{5}$ & $\mathrm{X}_{6}$ & $\mathrm{X}_{7}$ & $\mathrm{Y}_{1}$ & $\mathrm{Y}_{2}$ & $\mathrm{Y}_{3}$ & $\mathrm{Y}_{4}$ & $\mathrm{Y}_{5}$ \\
\hline 1 & $-1(5)$ & $-1(0)$ & $-1(1)$ & $-1(1)$ & $-1(1)$ & $-1(5)$ & $-1(25)$ & 3,90 & 0,07 & 0,042 & 0,13 & 0,003 \\
2 & $1(15)$ & $-1(0)$ & $-1(1)$ & $-1(1)$ & $1(5)$ & $-1(5)$ & $1(35)$ & 3,34 & 0,06 & 0,034 & 0,25 & 0,005 \\
3 & $-1(5)$ & $1(10)$ & $-1(1)$ & $-1(1)$ & $1(5)$ & $1(7)$ & $-1(25)$ & 4,12 & 0,10 & 0,042 & 0,09 & 0,001 \\
4 & $1(15)$ & $1(10)$ & $-1(1)$ & $-1(1)$ & $-1(1)$ & $1(7)$ & $1(35)$ & 4,04 & 0,08 & 0,042 & 0,66 & 0,022 \\
5 & $-1(5)$ & $-1(0)$ & $1(5)$ & $-1(1)$ & $1(5)$ & $1(7)$ & $1(35)$ & 3,93 & 0,16 & 0,181 & 0,63 & 0,009 \\
6 & $1(15)$ & $-1(0)$ & $1(5)$ & $-1(1)$ & $-1(1)$ & $1(7)$ & $-1(25)$ & 5,81 & 0,14 & 0,044 & 0,11 & 0,001 \\
7 & $-1(5)$ & $1(10)$ & $1(5)$ & $-1(1)$ & $-1(1)$ & $-1(5)$ & $1(35)$ & 3,94 & 0,07 & 0,213 & 0,88 & 0,022 \\
8 & $1(15)$ & $1(10)$ & $1(5)$ & $-1(1)$ & $1(5)$ & $-1(5)$ & $-1(25)$ & 6,52 & 0,14 & 0,032 & 0,45 & 0,012 \\
9 & $-1(5)$ & $-1(0)$ & $-1(1)$ & $1(5)$ & $-1(1)$ & $1(7)$ & $1(35)$ & 0,75 & 0,01 & 0,011 & 0,18 & 0,007 \\
10 & $1(15)$ & $-1(0)$ & $-1(1)$ & $1(5)$ & $1(5)$ & $1(7)$ & $-1(25)$ & 6,54 & 0,16 & 0,030 & 0,71 & 0,034 \\
11 & $-1(5)$ & $1(0)$ & $-1(1)$ & $1(5)$ & $1(5)$ & $-1(5)$ & $1(35)$ & 3,64 & 0,07 & 0,050 & 0,88 & 0,032 \\
12 & $1(15)$ & $1(10)$ & $-1(1)$ & $1(5)$ & $-1(1)$ & $-1(5)$ & $-1(25)$ & 7,53 & 0,15 & 0,043 & 0,78 & 0,024 \\
13 & $-1(5)$ & $-1(0)$ & $1(5)$ & $1(5)$ & $1(5)$ & $-1(5)$ & $-1(25)$ & 6,64 & 0,15 & 0,044 & 0,11 & 0,012 \\
14 & $1(15)$ & $-1(0)$ & $1(5)$ & $1(5)$ & $-1(1)$ & $-1(5)$ & $1(35)$ & 2,54 & 0,05 & 0,044 & 0,72 & 0,022 \\
15 & $-1(5)$ & $1(10)$ & $1(5)$ & $1(5)$ & $-1(1)$ & $1(7)$ & $-1(25)$ & 7,93 & 0,16 & 0,043 & 0,25 & 0,012 \\
16 & $1(15)$ & $1(10)$ & $1(5)$ & $1(5)$ & $1(5)$ & $1(7)$ & $1(35)$ & 5,32 & 0,09 & 0,102 & 0,99 & 0,024 \\
17 & $0(10)$ & $0(5)$ & $0(3)$ & $0(3)$ & $0(5)$ & $0(6)$ & $0(30)$ & 19,43 & 0,27 & 0,071 & 1,51 & 0,034 \\
18 & $0(10)$ & $0(5)$ & $0(3)$ & $0(3)$ & $0(5)$ & $0(6)$ & $0(30)$ & 19,51 & 0,27 & 0,071 & 1,52 & 0,034 \\
19 & $0(10)$ & $0(5)$ & $0(3)$ & $0(3)$ & $0(5)$ & $0(6)$ & $0(30)$ & 18,72 & 0,26 & 0,082 & 1,51 & 0,033 \\
20 & $0(10)$ & $0(5)$ & $0(3)$ & $0(3)$ & $0(5)$ & $0(6)$ & $0(30)$ & 19,34 & 0,27 & 0,071 & 1,52 & 0,034 \\
\hline
\end{tabular}

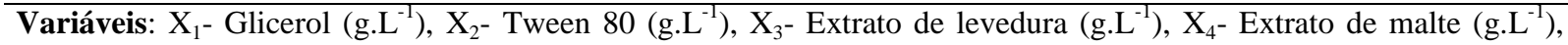
$\mathrm{X}_{5}$-Peptona (g.L $\left.\mathrm{L}^{-1}\right), \mathrm{X}_{6}-\mathrm{pH}$ e $\mathrm{X}_{7}$-Temperatura $\left({ }^{\circ} \mathrm{C}\right)$. Respostas: $\mathrm{Y}_{1^{-}} \mathrm{X}_{\max }\left(\mathrm{g} \cdot \mathrm{L}^{-1}\right), \mathrm{Y}_{2^{-}} \operatorname{Prod}_{\mathrm{X}}\left(\mathrm{g} \cdot \mathrm{L}^{-1} \cdot \mathrm{h}^{-1}\right), \mathrm{Y}_{3}-\mu_{\max }\left(\mathrm{h}^{-1}\right)$, $\mathrm{Y}_{4^{-}} \operatorname{Ativ}_{\max }\left(\mathrm{U}_{\mathrm{m}} \mathrm{mL}^{-1}\right)$ e $\mathrm{Y}_{5^{-}} \operatorname{Prod}_{\mathrm{E}}\left(\mathrm{U} \cdot \mathrm{mL}^{-1} \mathrm{~h}^{-1}\right)$

Por meio desse primeiro planejamento experimental fracionário, constataram-se melhores condições para o crescimento da levedura e para produção de lipase a condição do ponto central do planejamento (ensaios $17,18,19$ e 20). Tendo como objetivo principal do trabalho maximizar a atividade enzimática, foi realizada uma análise de efeitos das variáveis estudadas sobre a atividade lipolítica máxima (Tabela 4). 
Tabela 4 - Efeito das variáveis estudadas sobre atividade lipolítica máxima (Ativ ${ }_{\max }$ ).

\begin{tabular}{ccccc}
\hline Fator & Efeito $\left(\mathrm{U} \cdot \mathrm{mL}^{-1}\right)$ & $\mathrm{EP}$ & $\mathrm{t}(3)$ & $\mathrm{p}$ \\
\hline Media & 0,70 & 0,001 & 534,47 & $<0,001$ \\
Glicerol & 0,19 & 0,002 & 64,95 & $<0,001$ \\
Tween80 & 0,30 & 0,002 & 92,66 & $<0,001$ \\
Extrato de levedura & 0,05 & 0,002 & 16,45 & $<0,001$ \\
Extrato de malte & 0,18 & 0,002 & 64,08 & $<0,001$ \\
Peptona & 0,04 & 0,003 & 12,99 & $<0,001$ \\
pH & $-0,07$ & 0,003 & $-24,24$ & $<0,001$ \\
Temperatura & 0,32 & 0,003 & 112,58 & $<0,001$
\end{tabular}

EP- erro padrão CL- 90\%

Com base na análise de efeitos (Tabela 4), as variáveis $\mathrm{pH}$, peptona e extrato de levedura foram descartadas para o planejamento completo, pois apresentaram os menores efeitos sobre a atividade lipolítica. Com relação ao extrato de malte, este também foi descartado, pois se considerou que o aumento da sua concentração implicaria em custos adicionais e não compensaria o incremento observado na atividade lipolítica.

Tendo em vista que os melhores valores de atividade lipolítica se deram nas condições do ponto central do planejamento, para a sequencia do estudo, as concentrações de peptona, extrato de malte e extrato de levedura foram fixadas em 5,3 e 3 g.L. ${ }^{-1}$, respectivamente, enquanto que o $\mathrm{pH}$ foi fixado em 6,0. As variáveis selecionadas para o segundo planejamento experimental foram as concentrações de glicerol e Tween 80 e a temperatura.

\subsection{Planejamento experimental completo}

A Tabela 5 apresenta a matriz do planejamento experimental completo $2^{3}$ com 3 pontos centrais e 4 pontos axiais, totalizando 17 ensaios, visando à otimização da composição do meio de cultivo para a maximização da atividade lipolítica.

Nesse planejamento as variáveis independentes estudadas foram: a temperatura e as concentrações de glicerol e Tween 80. As respostas avaliadas foram: biomassa máxima $\mathrm{X}_{\max }\left(\mathrm{g} . \mathrm{L}^{-1}\right)$; produtividade em biomassa - $\operatorname{Prod}_{\mathrm{X}}\left(\mathrm{g} \cdot \mathrm{L}^{-1} \cdot \mathrm{h}^{-1}\right)$; velocidade especifica máxima de crescimento celular - $\mu_{\max }\left(\mathrm{h}^{-1}\right)$; atividade lipolítica máxima - Ativ $\max \left(\mathrm{U}_{\mathrm{mL}} \mathrm{m}^{-1}\right) \mathrm{e}$ produtividade enzimática $-\operatorname{Prod}_{\mathrm{E}}\left(\mathrm{U} \cdot \mathrm{mL}^{-1} \cdot \mathrm{h}^{-1}\right)$. 
Tabela 5 - Matriz do Planejamento completo $2^{3}$ e respostas obtidas

\begin{tabular}{ccccccccc}
\hline Ensaio & $\mathrm{X}_{1}$ & $\mathrm{X}_{2}$ & $\mathrm{X}_{3}$ & $\mathrm{Y}_{1}$ & $\mathrm{Y}_{2}$ & $\mathrm{Y}_{3}$ & $\mathrm{Y}_{4}$ & $\mathrm{Y}_{5}$ \\
\hline 1 & $-1(9,5)$ & $-1(4,5)$ & $-1(25)$ & 15,23 & 0,31 & 0,901 & 0,88 & 0,025 \\
2 & $-1(9,5)$ & $-1(4,5)$ & $+1(33)$ & 12,43 & 0,25 & 0,772 & 0,28 & 0,004 \\
3 & $-1(9,5)$ & $+1(10,5)$ & $-1(25)$ & 16,16 & 0,33 & 0,943 & 0,88 & 0,024 \\
4 & $-1(9,5)$ & $+1(10,5)$ & $+1(33)$ & 15,52 & 0,23 & 0,454 & 0,52 & 0,024 \\
5 & $+1(15,5)$ & $-1(4,5)$ & $-1(25)$ & 16,07 & 0,33 & 0,453 & 1,01 & 0,025 \\
6 & $+1(15,5)$ & $-1(4,5)$ & $+1(33)$ & 15,43 & 0,22 & 0,681 & 0,44 & 0,025 \\
7 & $+1(15,5)$ & $+1(10,5)$ & $-1(25)$ & 16,34 & 0,34 & 0,962 & 1,64 & 0,075 \\
8 & $+1(15,5)$ & $+1(10,5)$ & $+1(33)$ & 14,32 & 0,27 & 0,854 & 0,92 & 0,043 \\
9 & $0(12,5)$ & $0(7,5)$ & $-1,68(25)$ & 15,20 & 0,18 & 0,602 & 1,38 & 0,035 \\
10 & $0(12,5)$ & $0(7,5)$ & $+1,68(35)$ & 5,92 & 0,12 & 0,293 & 0,65 & 0,015 \\
11 & $0(12,5)$ & $-1,68(2,5)$ & $0(30)$ & 13,32 & 0,23 & 0,472 & 0,88 & 0,022 \\
12 & $0(12,5)$ & $+1,68(12,5)$ & $0(30)$ & 14,62 & 0,22 & 0,344 & 0,72 & 0,021 \\
13 & $-1,68(7,5)$ & $0(7,5)$ & $0(30)$ & 11,41 & 0,19 & 0,321 & 1,81 & 0,075 \\
14 & $+1,68(17,5)$ & $0(7,5)$ & $0(30)$ & 12,46 & 0,20 & 0,343 & 2,15 & 0,094 \\
15 & $0(12,5)$ & $0(7,5)$ & $0(30)$ & 12,05 & 0,22 & 0,331 & 1,75 & 0,045 \\
16 & $0(12,5)$ & $0(7,5)$ & $0(30)$ & 12,18 & 0,22 & 0,363 & 1,75 & 0,045 \\
17 & $0(12,5)$ & $0(7,5)$ & $0(30)$ & 12,22 & 0,21 & 0,344 & 1,85 & 0,054 \\
\hline
\end{tabular}

Variáveis: $\mathrm{X}_{1^{-}}$Glicerol (g.L $\left.\mathrm{L}^{-1}\right), \mathrm{X}_{2^{-}}$Tween $80\left(\right.$ g.L $\left.\mathrm{L}^{-1}\right), \mathrm{X}_{3^{-}}$Temperatura $\left({ }^{\circ} \mathrm{C}\right)$. Respostas: $\mathrm{Y}_{1^{-}} \mathrm{X}_{\max }\left(\mathrm{g} . \mathrm{L}^{-1}\right), \mathrm{Y}_{2^{-}}$Prod $_{\mathrm{X}}$ $\left(\mathrm{g} . \mathrm{L}^{-1} \cdot \mathrm{h}^{-1}\right), \mathrm{Y}_{3}-\mu_{\max }\left(\mathrm{h}^{-1}\right), \mathrm{Y}_{4}-\operatorname{Ativ}_{\max }\left(\mathrm{U}_{\mathrm{m}} \mathrm{mL}^{-1}\right)$ e $\mathrm{Y}_{5^{-}} \operatorname{Prod}_{\mathrm{E}}\left(\mathrm{U} \cdot \mathrm{mL}^{-1} \cdot \mathrm{h}^{-1}\right)$

Conforme mostrado na Tabela 5, os ensaios 13, 14, 15, 16 e 17 mostraram os melhores valores para atividade enzimática. Para todas as respostas estudadas foi feita análise de variância $(p \leq 0,05)$ para verificar a possibilidade de construção de um modelo empírico codificado, porém apenas para resposta atividade enzimática foi possível à construção de um modelo preditivo e significativo. A Equação 3 apresenta o modelo quadrático empírico para a atividade lipolítica máxima.

$$
\operatorname{Ativ}_{\max }\left(\mathrm{U} \cdot \mathrm{mL}^{-1}\right)=1,7-0,2(\mathrm{~T})-0,3\left(\mathrm{~T}^{2}\right)-0,4\left(\mathrm{~T}_{\mathrm{w}}{ }^{2}\right)+0,1(\mathrm{G})+0,1\left(\mathrm{~T}_{\mathrm{w}} \mathrm{G}\right)
$$

Sendo:

$\mathrm{T}$ - Temperatura $\left({ }^{\circ} \mathrm{C}\right)$

$\mathrm{T}_{\mathrm{w}}$ - Concentração de Tween $80\left(\mathrm{~g} . \mathrm{L}^{-1}\right)$

G - Concentração de glicerol (g.L $L^{-1}$ )

A partir do modelo empírico (Equação 3) foi possível obter as curvas de contorno (Figura 1) para a análise das melhores condições de temperatura, concentração de glicerol e Tween 80 para a atividade lipolítica. 


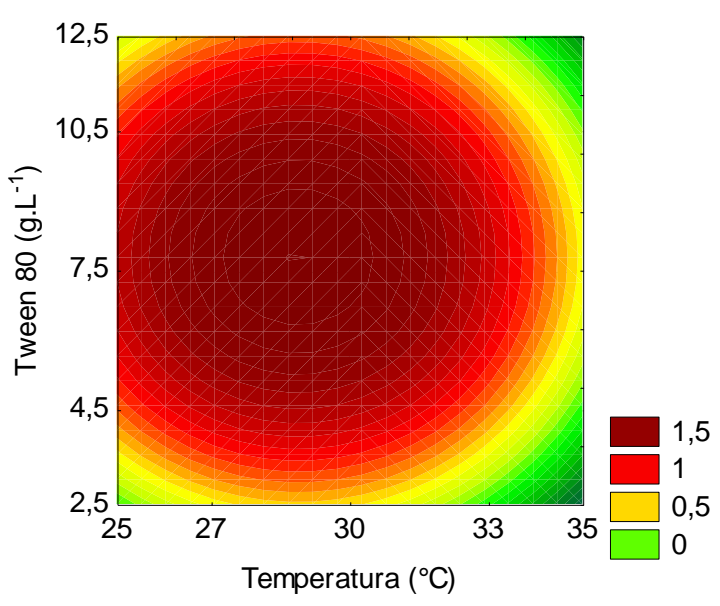

(a)

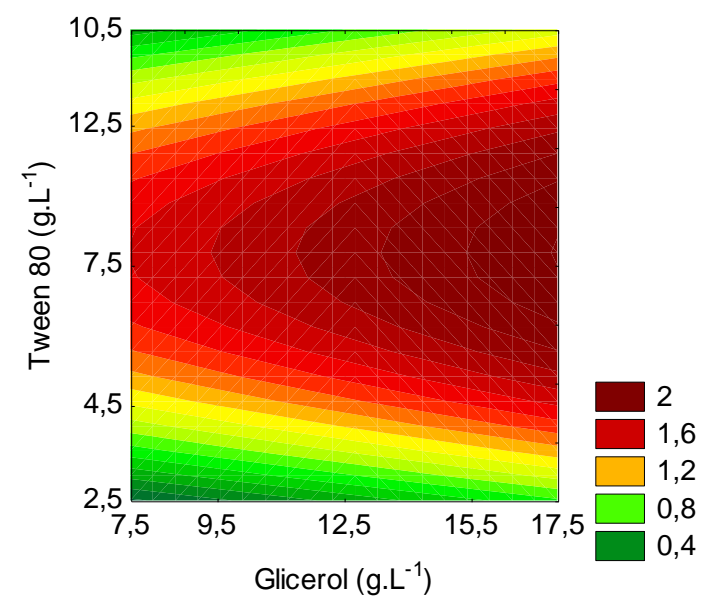

(b)

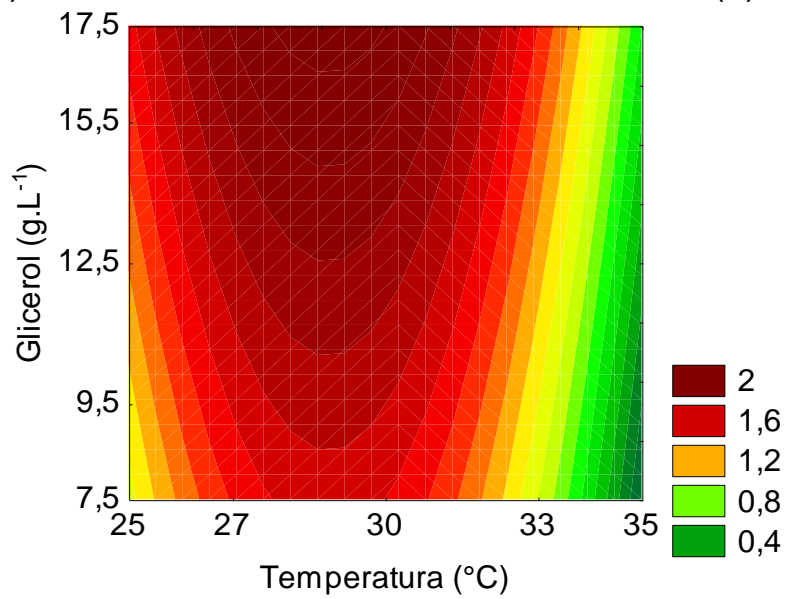

(c)

Figura 1- Curvas de contorno para atividade lipolítica em função de: (a) Tween 80 e temperatura $\left(\right.$ concentração de glicerol $=12,5$ g. $\left.\mathrm{L}^{-1}\right) ;\left(\right.$ b) Tween 80 e glicerol $\left(\right.$ Temperatura $\left.=30^{\circ} \mathrm{C}\right) ;(\mathrm{c})$ Concentração de glicerol e temperatura (concentração de Tween $80=7,5$ g.L $\mathrm{L}^{-1}$ ).

Dessa forma, como pode ser observado na Figura 1, verifica-se que a condição que possibilitou maior atividade enzimática corresponde a 17,5 g. $\mathrm{L}^{-1}$ de glicerol, 7,5 g.L $\mathrm{L}^{-1}$ de Tween 80 , temperatura de $30^{\circ} \mathrm{C}$, mantendo a concentração de $3 \mathrm{~g} . \mathrm{L}^{-1}$ para extrato de levedura e extrato de malte, $\mathrm{pH}$ 6,0 e $24 \mathrm{~h}$ de cultivo, o que resulta em uma atividade lipolítica de 2,15 U.mL $\mathrm{mL}^{-1}$, com uma biomassa máxima de $12,46 \mathrm{~g} . \mathrm{L}^{-1}$ produtividade de biomassa de 0,20 g. $\mathrm{L}^{-1} \cdot \mathrm{h}^{-1}$, velocidade especifica máxima de crescimento celular de $0,343 \mathrm{~h}^{-1}$ e produtividade enzimática de $0,094 \mathrm{U} \cdot \mathrm{mL}^{-1} \cdot \mathrm{h}^{-1}$.

Esses resultados são promissores com relação aos dados obtidos com o primeiro planejamento experimental, visto que se obteve um incremento de 2,8 vezes para a produtividade enzimática e 1,4 vezes para a atividade enzimática. 


\section{REFERÊNCIAS}

ACCARINI, J.H. Competitivo e sustentável. Revista Biodiesel Br., v. 12, n. 10, p.1020, 2009.

AOAC - ASSOCIATION OF OFFICIAL ANALYTICAL CHEMISTS. Official Methods of Analysis. $17^{\text {th }}$ edition. Washington, D.C., CD-ROM, 2000.

BILGEN, S.; KELES, S.; KAYGUSUZ, A.; SARI, A.; KAYGUSUZ, K. Renewable and sustainable energy reviews. Adv Biochem Eng Biot., v. 101, p. 372 - 396, 2008.

BURKERT, J.F.M. Otimização das condições de produção da lipase por Geotrichum candidum NRRL- Y552. Tese. Universidade Estadual de Campinas- UNICAMP, Campinas, Brasil, 2003.

HASAN, F.; SHAH, A. A.; HAMEED, A. Industrial applications of microbial lipases. Enzyme Microb Tech., v. 39, p. 235 - 251, 2006.

LEE, G.; JAE, H.; MIN, J. S.; IN-HWAN, K.; CHING, T. H.; HAK-RYUL, K. Optimal culture conditions for the production of a novel extracellular alkaline lipase from Yarrowia lipolytica NRRL Y-2178, J Microbial Biotech., v. 50, p.46 - 51, 2007

RIVALDI J.D.; SARROUH B. F.; SILVIO R. F.; SILVA S. Glicerol de biodiesel. Biotecnologia Ciência \& Desenvolvimento., v. 37, p. 44-52, 2007.

SILVA, G.P., MACK, M., CONTIERO, J. Glycerol: A promising and abundant carbon source for industrial microbiology. Biotech Advan., v. 27, p. 30-39, 2009.

TREICHEL, H.; OLIVEIRA, D.; MAZZUTI, M.A.; LUCCIO, M.D.; OLIVEIRA, V.J. A review on microbial lipase production. Food Bioprocess Tech., v. 3, p. 182-196, 2010. 